\title{
"Controlar" las Lecturas Literarias: Un estudio de casos sobre la Evaluación en el Plan de Lectura Complementaria de Educación Básica
}

\author{
To control the literary readings: a case study on the assessment of the complementary \\ reading plan in middle school \\ “Fiscalizar" as Leituras Literárias: Um Estudo de casos sobre a Avaliação no plano \\ de leitura complementar da Educação Básica
}

\author{
Felipe Munita y Maritza Pérez $^{\mathrm{b}}$
}

\begin{abstract}
${ }^{\text {a} E s c u e l a ~ d e ~ E d u c a c i o ́ n ~ B a ́ s i c a, ~ U n i v e r s i d a d ~ C a t o ́ l i c a ~ d e ~ T e m u c o ; ~ P r o g r a m a ~ d e ~ D o c t o r a d o ~ e n ~ D i d a ́ c t i c a ~ d e ~ l a ~}$ Lengua y la Literatura, Universidad Autónoma de Barcelona. Correo electrónico: fmunita@uct.cl

bFundación La Fuente; Programa de Máster de Investigación en Didáctica de la Lengua y la Literatura, Universidad Autónoma de Barcelona. Correo elecrrónico: mperez@fundacionlafuente.cl
\end{abstract}

\begin{abstract}
RESUMEN
El artículo presenta una investigación desarrollada en 2011 en Santiago de Chile: un estudio de casos sobre las evaluaciones que profesores de Segundo Ciclo de Educación Básica realizan para las lecturas literarias prescritas en el plan de lectura complementaria. El propósito consistió en indagar la relación entre las creencias y representaciones de los profesores sobre la lectura literaria y su forma de evaluarla en el plan lector de la asignatura de Lenguaje y Comunicación. Los resultados muestran una relación entre las prácticas evaluativas del docente y sus sistemas de creencias acerca de lo literario, así como una concepción predominantemente certificativa de la evaluación, que se manifiesta en los instrumentos evaluativos utilizados.
\end{abstract}

Palabras clave: lectura literaria, evaluación, creencias del profesor, plan de lectura complementaria.

\begin{abstract}
This article presents a research developed in 2011 in Santiago of Chile; a case study on the assessment that teachers of Middle School developed on books established by the Complementary Reading Plan. The main purpose was to investigate the relation between teachers' beliefs and representations of the literary reading and their way of evaluating the reading comprehension of their students in the subject of Language and Communication. The results show a relationship between teachers' evaluation practices and their systems of beliefs related to literature; as well as a predominant certifying conception of the evaluation, which is demonstrated by the instruments used in the assessments.
\end{abstract}

Key words: literary reading, assessment, teacher beliefs, complementary reading plan.

\section{RESUMO}

Apresenta-se pesquisa desenvolvida em 2011, em Santiago de Chile; um estudo de casos sobre as avaliações que professores do Segundo Ciclo da Educação Básica realizam para as leituras literárias prescritas no plano de leitura complementar. Indagou-se a relação entre crenças e representações de professores sobre a leitura literária e sua forma de avaliá-la no plano leitor do conteúdo Linguagem e Comunicação. Resultados mostraram uma relação entre as práticas avaliativas do docente e seus sistemas de crenças a respeito do literário, bem como uma concepção predominantemente certificativa da avaliação, que se manifesta nos instrumentos avaliativos utilizados.

Palavras chave: Leitura literária. Avaliação. Crenças do professor. Plano de leitura complementar. 


\section{DISEÑO DE LA INVESTIGACIÓN}

El origen de la investigación que a continuación presentamos atiende a una de las mayores problemáticas que, tradicionalmente, ha enfrentado la educación literaria en la escolaridad chilena. Esto es, la desmotivación que genera en la mayoría de los estudiantes el hecho, institucionalizado y convertido en rutina, de leer en forma obligada un libro para luego contestar una prueba sobre lo leído. Esta práctica es conocida en el país como "Plan de Lectura Complementaria" (en adelante, PLC), que no es otra cosa que un listado de novelas (muy minoritariamente volúmenes de cuentos u obras de teatro) prescritas para un nivel escolar determinado, que los estudiantes deben leer en forma obligatoria y que deviene en una instancia evaluativa, comúnmente una prueba escrita sobre el libro. La periodicidad de estas lecturas y evaluaciones suele ser mensual, pues se espera que a fin del año académico los estudiantes hayan leído entre 6 y 8 libros. Cabe señalar que la instancia descrita suele ser, para muchos de los estudiantes, su único contacto con el discurso literario en la Escuela Básica (y, para algunos, también en Educación Media). ${ }^{1}$

Así, surgió la idea de investigar más detenidamente las evaluaciones que se realizan en el marco del PLC, que suelen ser uno de los puntos más conflictivos en la relación de los estudiantes con la literatura. Y en ese contexto, nuestro propósito específico fue indagar en los motivos y razones que llevan al profesorado a realizar esas evaluaciones, viendo allí posibles conexiones con sus creencias acerca de la lectura literaria y de los aportes de esta en los procesos de aprendizaje de los estudiantes. Levantamos para ello la siguiente pregunta de investigación: ¿Qué tipo de relación se observa entre las creencias y representaciones de los profesores sobre la lectura literaria y su forma de evaluarla en el Plan de Lectura Complementaria?

Hemos enfocado el estudio en el $2^{\circ}$ Ciclo Básico, periodo escolar que atiende a niños y adolescentes de entre 10 y 14 años, mayoritariamente, pues creemos que representan niveles especialmente complejos en la relación de los estudiantes con la literatura: provenientes de un periodo de lectura literaria más libre y lúdico en los primeros cursos de Básica, es en estas edades cuando suele producirse el primer (y a veces definitivo) quiebre con el texto literario.

Los objetivos que orientaron el estudio fueron los siguientes:

- General: Comprender la relación existente entre las creencias y representaciones sobre la lectura literaria de los profesores de $2^{\circ}$ ciclo básico y la metodología utilizada para evaluarla en el Plan de Lectura Complementaria.

- Específicos: 1. Indagar en las creencias y representaciones de los profesores de $2^{\circ}$ ciclo básico sobre la lectura literaria; 2 . Analizar los instrumentos de evaluación utilizados por los docentes de $2^{\circ}$ ciclo básico para evaluar el Plan de Lectura Complementaria; 3. Relevar la concepción de evaluación que subyace a las prácticas evaluativas en el contexto del PLC de $2^{\circ}$ ciclo básico.

El actual sistema educativo chileno consta de 8 niveles de Educación Básica (Primaria) y 4 niveles de Educación Media (Secundaria), que conforman el total de los años de escolaridad obligatoria. A su vez, los 8 cursos de Educación Básica se agrupan en dos grandes ciclos: 1er Ciclo va de $1^{\circ}$ a $4^{\circ}$ año, mientras $2^{\circ}$ Ciclo corresponde a los cursos de $5^{\circ}$ a $8^{\circ}$ año básico. 
Para responder adecuadamente a la pregunta de investigación y a los objetivos formulados, se diseñó el estudio bajo un enfoque cualitativo, que buscaba comprender un determinado aspecto de la realidad a través de la aproximación interpretativa de los investigadores a los datos obtenidos.

La investigación se implementó en los meses de marzo y abril de 2011, en Santiago de Chile. La muestra considera 3 profesoras que se desempeñan en la asignatura de Lenguaje y Comunicación, en $2^{\circ}$ Ciclo Básico, en diferentes establecimientos educacionales de la ciudad, caracterizados por un alumnado de nivel socio-económico medio-bajo, dos de ellos, y de nivel medio, el tercero.

El instrumento de recogida de datos utilizado fue la entrevista semiestructurada. Además, se consideró una muestra de documentos escritos (pruebas) para enriquecer el análisis. De esta forma, podríamos contrastar las opiniones de primera fuente de las profesoras acerca de la lectura literaria y su evaluación, recogidas en las entrevistas individuales, con el corpus de instrumentos de evaluación que ellas utilizan para el PLC. La entrevista contó con un guión de base de cinco preguntas, que a su vez respondían a dos categorías de interés para nuestra investigación: las creencias y representaciones en torno a la lectura literaria y el enfoque de evaluación para el PLC. En ese marco, las preguntas son:

\section{1. ¿Cómo definiría usted la lectura literaria?}

2. Desde su perspectiva, ¿qué aporta el PLC a la educación literaria de sus estudiantes?

3. A su juicio, ¿por qué es importante evaluar la lectura de los libros del PLC?

4. ¿Qué instrumentos de evaluación utiliza usted para evaluar las lecturas del PLC?

5. ¿Qué criterios orientan la creación de estos instrumentos de evaluación?

Cabe señalar que con el sintagma "evaluar la lectura", que aparece ya en la tercera pregunta, remitíamos a la extendida práctica del profesorado sobre "controlar" los procesos de lectura de los estudiantes, es decir, certificar si una determinada lectura ha sido o no realizada, que es como suele entenderse en el vocabulario común del profesorado chileno. ${ }^{2}$ No obstante, elegimos la expresión evaluar la lectura pues, en un sentido procesual, podría remitir también a una instancia de regulación del proceso lector y de los aprendizajes que hay en juego, y nos propusimos averiguar si alguna de las participantes hacía esa relación en forma complementaria.

Además de la entrevista, cada una de las docentes facilitó 3 instrumentos evaluativos que ha utilizado en el PLC, los que sumados al registro de audio y a la transcripción de las unidades más significativas que realizamos de las entrevistas, nos permitieron contar con datos relevantes para responder a la pregunta formulada.

Es importante precisar que para el análisis de los datos provenientes de las entrevistas, desarrollamos un diseño emergente propio de la metodología cualitativa. Esto implicó realizar una codificación abierta de la información, para luego dar forma a un modelo comprensivo del fenómeno. En el caso de los instrumentos (pruebas escritas) el procedimiento utilizado fue el análisis de contenido, de manera tal que cada ítem o pregunta fue clasificado según los niveles de comprensión lectora que reseñaremos más adelante.

2 No es casualidad que, en este contexto, el instrumento evaluativo que se aplica en el PLC sea conocido como "Control de lectura". 
En una primera instancia, cada investigador realizó un análisis individual de los datos, para luego triangular los resultados con el(la) co-investigador(a).

De nuestra pregunta de investigación se desprenden tres ámbitos a los que es necesario atender en el marco de referencia: la evaluación en el área de lengua y literatura, la concepción de lectura literaria y su evaluación en el marco de la comprensión lectora $\mathrm{y}$, finalmente, los sistemas de creencias y representaciones del profesorado.

\section{MARCO DE REFERENCIA}

\subsection{LA EVALUACIÓN EN EL ÁREA DE LENGUA Y LITERATURA}

Actualmente, hay consenso en señalar que la evaluación constituye uno de los ejes del currículum en cualquiera de las disciplinas escolares y que las funciones que cumple en el proceso de enseñanza y aprendizaje son diversas y corresponden a múltiples propósitos. Los avances de la investigación en este campo nos presentan en forma organizada esta diversidad de funciones. Allal (2006), a partir de las propuestas de Cardinet y de Bloom, diferencia las funciones de regulación, certificación y orientación que podrían emparentarse con los tipos de evaluación formativa, sumativa y predictiva. ${ }^{3}$ En este marco, se ha entendido la evaluación como una serie de instancias que, integradas en el proceso de aprendizaje, cumplen con dos finalidades claramente diferenciadas: por una parte, conocer qué y cómo aprenden los alumnos, para así poder ayudarles y, por otra, certificar el resultado de los aprendizajes obtenidos (Ribas, 2010).

En el área de lengua y literatura, las actuales propuestas evaluativas (dentro del ámbito de la que hemos llamado "formativa") están centradas en los procesos de mejora de las competencias comunicativas de los estudiantes y, particularmente, en las habilidades de lectura, se ha concebido la evaluación como un instrumento "no solo para ponderar hasta qué punto el proceso de interpretación ha tenido lugar sino y, especialmente, para afianzar este proceso de comprensión o interpretación y para colaborar en él. Estaríamos entonces frente a una evaluación entendida como una mediación" (Sánchez Miguel, 1997: 83). ${ }^{4}$ En otras palabras: alejándose del tradicional sentido de "certificación" de un nivel de lectura o de "comprobación" acerca de si una lectura ha sido o no realizada, limitadas únicamente a una función sumativa, en la actualidad se busca que la práctica evaluativa se transforme en un proceso de ayuda para la progresiva adquisición de la competencia de lectura, sumando así las perspectivas de regulación y orientación antes mencionadas.

Esta concepción de la evaluación ha supuesto, en los últimos años, un creciente interés por el concepto de "evaluación formativa", entendida en nuestra disciplina como un "instrumento al servicio de la autorregulación metacognitiva del alumno", que permite "ejercer un control sobre el propio aprendizaje y sobre los procesos de producción y comprensión lingüísticos" (Ribas, 1997: 6). Así, el progreso de la competencia comunicativa del estudiante se asocia, en buena medida, a la reflexión que este sea capaz

3 Aunque la investigadora de la Universidad de Ginebra asocia la función reguladora a todo tipo de evaluación, no sólo a la formativa.

4 Las citas de textos originalmente escritos en catalán y en francés han sido traducidas por los autores de este artículo, para facilitar su lectura. 
de realizar sobre los fenómenos lingüísticos y literarios trabajados, reflexión inducida mediante instancias evaluativas que permiten al alumno observar el proceso productivo e interpretativo que lo ha llevado a conseguir determinados "productos" lingüísticos, y objetivar dicho proceso mediante un ejercicio de autorregulación metacognitiva.

Los avances presentados por la investigación, sumados a la idea de la evaluación como "eje vertebrador" del currículum, han llevado a Briz Villanueva (1998, 2003) a proponer un modelo evaluativo para el área de lengua y literatura que considera la evaluación como un proceso de cohesión y coherencia, en el que:

...se produce una planificación consciente y sistemática del proceso evaluador en el marco de una estructura definida por una concepción ideológica, ética y curricular con unos objetivos y funciones determinados que se desarrollan a lo largo del tiempo en un contexto concreto, en virtud de la cual es preciso seleccionar y coordinar las estrategias, los medios e instrumentos necesarios (2003: 431).

Es decir, la evaluación como un dispositivo de coherencia entre los objetivos perseguidos, las actividades planificadas, los instrumentos y procedimientos seleccionados, las concepciones del enseñante sobre los saberes que están juego y todo en el marco de un contexto de enseñanza-aprendizaje determinado, que precisa y le da sentido a la interrelación entre los diversos factores considerados. La relevancia que cobra aquí la pregunta acerca de las destrezas que queremos evaluar es evidente: la coherencia en la evaluación de las habilidades lingüísticas implica, en primer lugar, definir la naturaleza del objeto, es decir, qué evaluar (Briz Villanueva, 2003) y, a partir de ahí, buscar los mecanismos y procedimientos que colaboren de mejor manera en la consecución de los objetivos trazados. En palabras de Teresa Ribas:

Si queremos que la evaluación tenga incidencia en el proceso formativo, es bueno que identifiquemos el objeto que estamos evaluando en cada momento. Evidentemente, la forma de conceptualizar el objeto determinará las informaciones que se recojan para la evaluación (2010: 13).

Lo anterior nos lleva a la pregunta por el objeto de evaluación, que en el marco del presente estudio se circunscribe a la lectura literaria, para observar cuáles son los objetivos y criterios que la investigación ha relevado como claves para la adquisición de la competencia específica que implica la lectura de textos literarios y, posteriormente, para indagar los objetivos que el profesorado tiene a la vista a la hora de evaluar las lecturas literarias que prescribe.

\subsection{LECTURA LITERARIA Y EVALUACIÓN DE LA COMPRENSIÓN}

Luego de un largo periodo marcado por las formulaciones propuestas desde el formalismo y el estructuralismo, definidas en función de un análisis únicamente textual y centradas en aquellos aspectos de "literariedad" que distinguían a la literatura en relación a otras formas discursivas, en las últimas décadas hemos asistido a un progresivo cambio de paradigma en cuanto a la definición de "literatura" y, en particular, de lo que significa "leer literatura". Anclados fundamentalmente en los aportes de la Estética de la Recepción, de la Pragmática y de la Semiótica literaria, estos cambios han implicado un desplazamiento del interés puramente textual hacia el diálogo que establece el lector con el texto en una situación comunicativa determinada. 
"La concepción actual de la lectura supone la consideración establecida por muchos autores de cómo se interrelacionan tres factores: el lector, el texto y el contexto de lectura" (Colomer, 1998: 52). Esta concepción, formulada en términos genéricos para todo tipo de lectura, adquiere nuevos sentidos en el discurso literario, dada la ambigüedad poética, la polisemia y las virtualidades expresivas que lo caracterizan. Así, en la lectura literaria, entendida como un "acto de creación estética", "lo decisivo es lo que ocurre en nosotros mismos como consecuencia de haber leído el texto, según viene poniendo de manifiesto la poética de la lectura" (Sánchez Corral, 2003: 297). Allí, por tanto, debieran apuntar las prácticas didácticas (y, por extensión, las prácticas evaluativas) para colaborar en la formación de una competencia literaria definida, en buena medida, por la actividad interpretativa del lector.

En este contexto, la investigación educativa ha establecido ciertos criterios para el trabajo con los libros de literatura (Colomer 2005, a partir de Cairney, 1992), entre los cuales destacaremos los siguientes:

1. Proponer análisis centrados en el significado global y no en detalles irrelevantes. ${ }^{5}$

2. Tareas de tipo "abierto" (no de respuesta única) que susciten la reflexión sobre lo leído.

3. Tareas electivas, según la consonancia con el diálogo de cada individuo con el texto.

4. Propuestas de trabajo cooperativo, que posibiliten la interpretación y construcción conjunta de significados.

Como puede observarse, los criterios avanzan en la perspectiva de la poética de la lectura, hacia la focalización en la implicación subjetiva del lector y, sobre todo, en la construcción de significados que este realiza a partir del texto (desde el texto, pero no únicamente en el texto). En la misma línea, otros autores han postulado las discusiones y debates entre los compañeros, las informaciones provenientes del mediador y las referencias intertextuales a las obras ya leídas, como los principios orientadores al momento de programar las actividades de lectura literaria en el aula (Sánchez Corral, 2003), han destacado la implicación personal y el diálogo sobre las obras como elementos imprescindibles para el desarrollo de actitudes favorables hacia la literatura (Bordons \& Díaz Plaja, 2004) y han señalado que las innovaciones en la investigación sobre la lectura apuntan al protagonismo del lector en la construcción del significado y a las ayudas que podamos darle para la elaboración de respuestas personales más complejas (Colomer, 1996).

En este entramado de criterios y propuestas de lineamientos didácticos, coherentes con la concepción actual de la lectura literaria, debemos situar las prácticas escolares de evaluación de la comprensión lectora, para indagar así en el grado de consonancia que manifiestan frente a los avances producidos en la investigación.

Colomer y Margallo (s/f) hacen una síntesis de las principales taxonomías que se han postulado para clasificar los tipos de preguntas de comprensión de lectura, destacando

5 Especialmente interesante es la propuesta de Sánchez Miguel (1997) al respecto, que resalta la importancia de "dirigir la mente del alumno hacia el significado global del texto" como primer principio orientador para la evaluación de la comprensión lectora. 
la de Bloom y la de Pearson y Johnson como las más influyentes. La primera considera preguntas de reconocimiento literal (o recuerdo), inferencias y preguntas de evaluación y de apreciación. La segunda se basa en una diferenciación según tres niveles en la relación pregunta-respuesta: de relación explícita y textual, de relación implícita y textual (inferencias) y de relación implícita y fundamentada sobre esquemas del lector. Podríamos considerar entonces, como tradicionalmente se ha hecho, tres grandes niveles que definen las preguntas de comprensión de los textos: literal, inferencial y crítico o apreciativo, que lejos de ser "etapas consecutivas" en la adquisición de la competencia literaria, corresponden a los diferentes grados de profundización de una lectura determinada.

Ahora bien, diversas investigaciones realizadas en este ámbito han observado qué tipos de preguntas se realizan comúnmente en las escuelas a la hora de evaluar la comprensión lectora. Colomer recoge estas observaciones alertando que los resultados muestran "que las actividades se basan en la comprensión literal y que las lecturas se evalúan más que guían" (2009: 10). En una línea similar, Sánchez Miguel (1997) nos previene sobre cómo suele sobreentenderse que comprender equivale a contestar el mayor número posible de preguntas sobre lo que trata el texto. Igualmente Tauveron (2002), que desde el contexto escolar francés muestra cómo los manuales estándar hacen preguntas de "comprensión", cuyo interés por una verdadera comprensión es a lo menos discutible. La autora demuestra que muchas de las propuestas solo implican una actividad primaria de "cortar/ pegar", y que las preguntas sobre la apreciación del lector (acercamiento lógico y esperable en la perspectiva de la poética de la lectura) son muy poco frecuentes. Reflexionando sobre cómo leemos comúnmente la literatura en la escuela, Tauveron señala:

Verificamos su "comprensión" por el mismo tipo de cuestionario que usamos para todos los demás escritos, un cuestionario centrado primero sobre el nivel literal del texto. No ponemos en juego, concerniéndole, ninguna postura particular de lectura. La atención se lleva casi exclusivamente hacia "lo que se cuenta" (la fábula o la intriga) en una lectura referencial que deja poco espacio a la dimensión simbólica y estética (2002: 14).

Por tanto, se ha visto que a la hora de leer literatura igualmente predomina un tipo de lectura referencial, con un marcado énfasis en la literalidad del texto y que queda en tela de juicio al contrastarlo con los objetivos y criterios didácticos asociados a la educación literaria: ¿en qué medida este tipo de preguntas son efectivamente una ayuda cuando no integran -o lo hacen levemente- el trabajo interpretativo del alumno? Pues pareciera que la formulación de preguntas sobre la comprensión del texto sigue en la perspectiva que Tauveron ha denominado como "estatus de la evidencia", concepto que no nos lleva sino a pensar en ejercicios que han "servido únicamente para evaluar si los niños han sido capaces de hacerlos" (Colomer, 2005: 95).

En definitiva: si atendemos a las observaciones realizadas por la investigación en diversos contextos (catalán, español y francés, al menos), podemos decir que el panorama general de la comprensión lectora en la escuela continúa centrado en la búsqueda del significado literal del texto, situación mucho más afín con las formulaciones de la poética del texto que con los avances realizados en la teoría literaria y en la didáctica de literatura de las últimas décadas. 


\subsection{EL PENSAMIENTO DEL PROFESOR: CREENCIAS EN EL CONTEXTO DE LA EDUCACIÓN LITERARIA}

La propuesta central del artículo de Sánchez Miguel aludido podría sintetizarse como sigue: la evaluación de la capacidad de comprensión de un estudiante depende de la concepción del profesor sobre lo que es un texto, en nuestro caso un texto literario, y del papel que este concede al proceso para comprenderlo. Esto nos lleva, pues, a indagar en los sistemas de creencias del profesorado y en su incidencia en las prácticas didácticas que promueve en el aula, especialmente en las referidas a la evaluación de la lectura literaria.

La idea de base para las investigaciones sobre el pensamiento del profesor considera que toda actuación didáctica está condicionada, en buena medida, por las ideas que el docente tiene acerca del objeto de enseñanza y del proceso de aprendizaje que este implica. En palabras de Margarida Cambra: "El profesor no se apoya simplemente en unos conocimientos teóricos y procedimentales adquiridos durante los periodos de formación, sino que filtra el contenido de los programas de formación a través de su sistema personal de creencias, saberes y representaciones" (2000: 161).

En esta línea, la investigación educativa actual ha definido un modelo basado en los conceptos de creencias, representaciones y saberes (Cambra, 2000) que actúa como marco de referencia tanto para los discursos del profesorado en un determinado ámbito como, sobre todo, para las prácticas didácticas que selecciona e implementa en el contexto del aula. En este modelo, Cambra plantea las siguientes diferencias entre los saberes y las creencias y representaciones: mientras estas son proposiciones cognitivas no necesariamente estructuradas (individuales las creencias, sociales las representaciones), aquellos son estructuras cognitivas esquemáticas y definidas convencionalmente, referidas a aspectos determinados de un área disciplinar y a los procesos de enseñanza y aprendizaje asociados al aspecto disciplinar en cuestión. En este marco, cabe señalar que las creencias y representaciones se han observado como muy influenciadas por las experiencias que el docente haya tenido como aprendiz en su propia etapa de escolaridad (y también en su formación universitaria); estas experiencias constituyen un marco de actuación que reaparece de diversas maneras en su práctica profesional.

En definitiva, la interrelación entre los conocimientos que el docente ha adquirido en las diferentes instancias de formación en las que ha participado, y las ideas y concepciones que tiene acerca del proceso de enseñanza-aprendizaje, del objeto de ese proceso e, incluso, de los estudiantes con quienes trabaja, forman un entramado desde el cual el docente selecciona unas determinadas estrategias y procedimientos que orientan y definen su práctica profesional.

Llevado esto al ámbito de lengua y literatura y, en particular a la adquisición de la competencia de lectura literaria por parte de los estudiantes, pasa a ser relevante la pregunta por las concepciones que el docente tiene de la lectura literaria y de la funcionalidad de la evaluación de la lectura de obras prescritas en el contexto del desarrollo de la competencia literaria de sus alumnos:

La concepción personal de la materia, del enfoque, así como el grado de implicación en la concepción metodológica y la forma de aplicación, junto con las creencias del profesor sobre la funcionalidad de la evaluación, constituyen el bloque de factores variables que afectan los procedimientos y los recursos aplicados a la evaluación (Mendoza, 2000: 131). 
Aceptada esta idea, resulta evidente que los instrumentos y recursos de evaluación que utiliza el docente están mediados también por sus representaciones y creencias, compartidas en la cultura profesional del profesorado, aquellas individuales y personales, estas. Y ambas actuarían como "sistemas de teorías implícitas" (Palou et al., 2000) que ayudarían a explicar muchas de las decisiones que toma el docente en el aula, entre otras, su forma de evaluar las obras literarias de prescripción escolar.

\section{ANÁLISIS DE DATOS Y DISCUSIÓN DE LOS RESULTADOS}

Para realizar el análisis de la información recopilada hemos conservado las categorías operativas creadas para la construcción de la pauta de entrevista. De esta manera, comenzaremos por describir las principales creencias y representaciones surgidas en torno a la lectura literaria, para luego referirnos al enfoque evaluativo adoptado por las docentes a la hora de evaluar las lecturas del Plan de Lectura Complementaria (PLC). Posteriormente, contrastaremos estos resultados con los datos provenientes del análisis de los instrumentos evaluativos utilizados por las profesoras.

\subsection{CREENCIAS Y REPRESENTACIONES SOBRE LA LECTURA LITERARIA ${ }^{6}$}

Hay que precisar que frente a la pregunta por la definición de la lectura literaria, no todas las docentes fueron capaces de elaborar un discurso satisfactorio y, en todos los casos, se debió plantear la pregunta varias veces y de distintas formas, con el fin de ampliar las respuestas y así evitar que estas se redujeran a una categorización netamente escolar. Aún así, en un caso en particular, el de Ana, ${ }^{7}$ no se logró una mayor elaboración sobre la definición de este tipo de lectura, pues sus respuestas se limitaron a conceptualizar la lectura literaria considerando su mayor extensión respecto a otro tipo de textos, como, por ejemplo, las lecturas incluidas en el libro de texto de la asignatura de Lenguaje, muchas de las cuales son también lecturas literarias. Por tanto, Ana evidencia una pobreza conceptual que la lleva, en definitiva, al desconocimiento de lo que se considera como "literatura" (cuyo rasgo distintivo está lejos de ser la extensión de los textos) y de aquello que implica "leer literatura":

Son textos más completos, más largos, más estructurados... uno puede elegir los temas específicos que quiere entregar de acuerdo a la edad de los niños (Ana).

Entre quienes sí lograron desarrollar un poco más el concepto (Teresa, Carmen) surgieron nociones relacionadas con la ampliación del conocimiento, el desarrollo del pensamiento, el incentivo de la imaginación y la creatividad y un cierto contacto con la belleza del texto. A continuación, algunas citas ilustrativas:

Más que nada ampliar su conocimiento en base a la belleza que tiene la lectura literaria. A conocer más el mundo literario en sí, que es muy diferente a lo que ellos están acostumbrados a ver, que es más que nada películas, tv, series (Teresa).

\footnotetext{
6 Cabe señalar que, para efectos del análisis, no haremos la distinción entre concepciones individuales de cada docente (sus creencias) y concepciones compartidas por el sistema cultural del profesorado (las representaciones), pues esto implicaría extenderse en una línea de análisis que escapa a los límites del presente trabajo.

7 Utilizamos nombres ficticios para anonimizar los datos.
} 
...claramente hay ciertos aspectos que la lectura literaria desarrolla o incentiva que la no literaria no. Esto es la imaginación, la creatividad, cosas que dentro de una noticia o una receta no puedes explorar (Carmen).

En ambos casos, se evidencia una concepción de la literatura como un tipo de discurso particular y con características propias, aunque no está muy claro en qué radica su especificidad. Así, mientras Teresa recurre a una lógica basada en el soporte textual, pues para hablar de la diferencia de la literatura hace una comparación con la ficción audiovisual, Carmen recurre a las habilidades que esta desarrollaría en el lector, habilidades sin duda menos promovidas en el contacto con textos funcionales. En este marco, resulta especialmente interesante la mención de Teresa en relación a la "belleza" de los textos, que establece ciertas relaciones implícitas con la manipulación estética del lenguaje, la connotación y la poeticidad que definen lo literario, aun cuando no profundiza en los elementos que desde su visión configurarían esa belleza.

En términos generales: de lo descrito anteriormente podemos inferir que las docentes no cuentan con un discurso fundamentado conceptualmente acerca de lo que significa la lectura de textos literarios. Pues incluso las ideas que avanzan en la dirección correcta, esto es, la "belleza" de Teresa y la "creatividad e imaginación" de Carmen, lo hacen sin fundamentarse o basándose en un discurso estándar correspondiente a las representaciones sociales construidas sobre el hecho literario. Incluso, las dudas que generó la pregunta dan cuenta de que, al parecer, tampoco hay una reflexión consciente acerca de la especificidad de lo literario en el marco de los diversos géneros discursivos de una sociedad.

Ahora bien, al dirigir las indagaciones hacia el ámbito específico del rol que cumpliría el PLC en la educación literaria de los estudiantes, las entrevistadas coinciden en atribuirle dos funciones principales: (1) generar hábitos de lectura, es decir, la costumbre por leer; y (2) dar oportunidad de frecuentación de los libros, es decir, el acceso a los materiales de lectura. Las siguientes citas así lo reflejan:

...en la actualidad en las casas no hay libros. Antiguamente uno se criaba entre libros o diarios, revistas. Siempre había algo que leer, ahora no. Entonces hay padres que compran exclusivamente los libros que le piden en el colegio a los niños, si no, no leerían, leerían solamente lo que leen en las horas de clases (Ana).

El hábito de la lectura diaria, más allá de lo que nosotros podemos leer (...) pero eso de manejar un libro, que ellos si van en el metro lean, aunque sea porque tienen que leerlo conmigo, en el velador manejar el texto porque en cualquier minuto pueden tomar el texto y leer y esa costumbre sólo se hace acá, es difícil que después (Carmen).

Yo creo que su crecimiento personal en cuanto a lo que ellos pueden ir logrando e incentivándoles la lectura. La idea es que no llegue a ser obligatoria, y que después sea por gusto. $Y$ que puedan ir solos, ya sea a la bibliometro o conseguirse un libro o de repente si voy al mall o la feria poder tentarme con un libro -aunque sea por el título del libro- y comprarlo, aunque lean el inicio, pero ya el tenerlo es como distinto (Teresa).

$\mathrm{Al}$ contrastar las citas con las ideas respecto a la lectura literaria expuestas en el marco de referencia, es posible señalar que en ninguno de los casos el discurso elaborado refleja la adopción de una perspectiva que propugne el encuentro y diálogo entre el lector y las obras literarias, ni que apunte a la idea de desarrollar la capacidad interpretativa del lector. Tampoco se espera que el PLC sea un aporte en términos del aprendizaje de 
las convenciones literarias, ni en el progreso de las habilidades lectoras de los estudiantes, ni en la ampliación -y progresiva complejización- del corpus de lectura (Colomer, 2005), cuestiones todas que parecieran no corresponder a su ámbito de desarrollo. De esta manera, la función del PLC se agota en sus posibilidades de frecuentación de la lectura por parte de los estudiantes y en un hipotético potencial para el desarrollo de hábitos lectores estables.

En ese contexto, es el discurso de Teresa el que incorpora las ideas más interesantes, como un aporte de las lecturas en términos del crecimiento personal y un desarrollo progresivo del gusto, ideas que no aparecen en las respuestas de las otras entrevistadas. Nos parece importante evidenciar el surgimiento de estas ideas que denotan una mirada más amplia de los propósitos que debieran guiar un plan de lecturas, ya que eventualmente estas permitirían observar algunas diferencias a la hora de relacionarlas con los enfoques de evaluación adoptados para evaluar el PLC.

\subsection{ENFOQUE DE EVALUACIÓN EN EL PLC}

Para avanzar hacia el terreno de la evaluación, en primer lugar se les consultó a las docentes por la importancia que tiene la actividad de evaluación de las lecturas de los libros prescritos en el PLC. En sus respuestas, se advierte la preponderancia que adquiere la idea de certificación de los aprendizajes y verificación de que se ha leído, concediéndole de esta manera una función principalmente sumativa a la evaluación de las lecturas. En los extractos que siguen es posible observar lo anterior:

Para ver si realmente entendieron los temas, los objetivos que tú querías lograr. Y también para incentivar las habilidades... las inferencias, las valóricas (Ana).

Nosotros estamos obligados a evaluarlo. No es que yo quiera o no, por mí, yo no pondría nota en nada, porque siempre he considerado que... o sea, el sistema te lo exige porque cómo voy a promover a un niño... pero a lo mejor si fuera sin nota pienso que sería mejor... en algunos niños más responsables... pero como estoy obligada a evaluarlos, tengo que evaluarlos. Es una lectura mensual mínima (Teresa).

Para saber si realmente leyeron el libro, porque los niños se van a los resúmenes. El niño que se va a un resumen, no tiene imaginación ni siquiera para hacer un dibujo. Y para ver su aprendizaje, hasta dónde llegaron. Y el sistema te exige además, poner una nota. Pero es más que nada eso, para evaluar los aprendizajes y para ver lo que ellos realmente lograron con la lectura (Teresa).

Sí, claro, porque yo tengo un objetivo con eso y yo tengo que evaluar ese objetivo... porque me está sirviendo para algo (...) además que te sirve para diagnosticar un montón de otras cosas... por ejemplo, a lo mejor al niñito no le cuesta leer los cuentos cortitos de la guía, pero sí tiene problemas con una historia más larga, o sí tiene problemas para identificar características físicas porque las confunde con las psicológicas por ejemplo. Entonces hay un montón de cosas que tú necesitas, un feedback, porque si no, no sirve para nada. Ahora otra cosa es que a lo mejor el profesor que esté efectivamente (...) no lo va a considerar como nota, pero aunque sea una evaluación cognitiva, evaluación tiene que hacer. Tú necesitas esa información. Yo en mi caso sí les pongo nota (Carmen).

Observamos que en las citas de Carmen y Teresa, además del concepto de certificación, se alude a otras nociones sobre las cuales es interesante detenerse. En el discurso 
de Carmen, por ejemplo, es posible hallar una referencia al concepto de diagnóstico. Según plantea, una de las razones para evaluar la lectura tiene que ver con la posibilidad de "diagnosticar un montón de otras cosas", y así obtener una especie de feedback respecto a los aprendizajes alcanzados por sus estudiantes y a las habilidades de lectura que presentan en un momento determinado. En sus palabras podríamos vislumbrar el esbozo de una concepción orientada hacia la evaluación formativa, al considerarla una instancia para conocer qué y cómo aprenden sus alumnos y así poder ayudarles. Sin embargo, a nuestro juicio, esta idea no llega a realizarse en su discurso, ya que se limita a las posibilidades del docente de contar con información que le permita retroalimentar a los estudiantes, sin considerarlos a ellos mismos dentro de este proceso de reorientación. Vale decir, no se contempla en ese diagnóstico a los estudiantes como sujetos capaces de autoregular y controlar metacognitivamente sus aprendizajes, tal como plantea Ribas (1997) al referirse a la evaluación formativa.

Por su parte, Teresa plantea la importancia de evaluar estas lecturas en base a dos razones: para evaluar los aprendizajes logrados y para responder a una exigencia del sistema educativo. Sobre esto último, en sus palabras se aprecia un cierto cuestionamiento respecto a la obligación de evaluar las lecturas. Según plantea, si estuviera dentro de sus posibilidades no calificaría con una nota las lecturas del PLC, en especial en el caso de los estudiantes considerados responsables. No obstante, en su discurso no se logran advertir las razones que sustentan esta postura, ya que sólo se limita a señalar que "sería mejor" sin profundizar más en la idea. ${ }^{8}$ Finalmente, al avanzar en su respuesta, Teresa se decanta por un argumento ligado explícitamente a la función certificativa de la evaluación de la lectura.

Al indagar en el tipo de instrumentos de evaluación utilizados en el PLC, se advierte que todas las entrevistadas coinciden en señalar la prueba escrita como principal herramienta evaluativa. A continuación, algunas citas que lo confirman:

Generalmente se usa la prueba escrita. Hay veces que se puede usar también el trabajo grupal, confección de historietas, pero generalmente se usa la evaluación. El niño está acostumbrado a la prueba escrita (Ana).

Yo hago pruebas. Pruebas objetivas con preguntas abiertas y de selección múltiple, donde incluye términos pareados, completación y todo eso (Teresa).

En casi su totalidad son con pruebas las lecturas, y que yo voy viendo distintos tipos de habilidades dentro de la prueba. Por ejemplo, la primera parte es de "completo", donde el niño... la información necesaria, después "respondo" que es como desarrollo, después una parte de opinión donde el niño manifiesta su opinión, otra de deducción o conclusión o inferencia a veces, depende, y otra de relato donde tiene que contar. Entonces ahí uno ve como está el niño en descripción, en opinión, en... es toda una gama, hay algunas en que agrego orden cronológico. Por lo general esos son los ítems que tienen mis pruebas (Carmen).

La prueba escrita, por tanto, aparece como la opción más recurrente para evaluar las lecturas. No obstante, las entrevistadas señalan haber implementado en alguna ocasión otras estrategias de evaluación, tales como exposiciones (en power point o papelógrafos), dramatizaciones, confección de historietas, entre otras:

8 Si bien esta situación puede originarse en que aún no tenga la idea bien formulada, puede deberse también a que simplemente no encontró cabida para ello en el desarrollo de la entrevista. 
Además en algunos casos he hecho representar ciertas partes del libro, tipo dramatización. Me acuerdo que el libro "¿Quién se ha llevado mi queso?" (...) me fui a evaluarlo a Rancagua, donde ellos hicieron laberintos, todo con papel... y fueron haciendo todo un cuento, en grupos. Salió todo espectacular, les encantó. Pero, para todo eso necesitas tiempo y recursos. Con ilustraciones, yo igual hago que... siempre en la prueba, aunque esa parte no vaya evaluada, pero le pongo: ilustre una parte del cuento y explíquela, para ver hasta dónde va su imaginación, cómo se imagino el huevito, el perrito o el personaje (Teresa).

A final de año es la única en que ellos hacen power point por ejemplo o hay veces que se disfrazan de los personajes, o hacen un papelógrafo, ahí ellos tienen que buscar una manera de... (Carmen).

Generalmente se usa la prueba escrita. Hay veces que se puede usar también el trabajo grupal, confección de historietas, pero generalmente se usa la evaluación (Ana).

Tal como se puede apreciar, el uso de otras herramientas de evaluación es de carácter esporádico y circunstancial: por ejemplo, el último libro del año. De acuerdo a las palabras de Teresa, los motivos para no implementar de manera más sistemática este tipo de estrategias responden a la falta de tiempo y recursos, dos aspectos que suelen aparecer como argumentos recurrentes en el discurso del profesorado frente a la innovación de sus prácticas didácticas (y que no necesariamente son piedras de tope para ello, como han demostrado muchas experiencias pedagógicas exitosas).

Ahora, si se revisa con cuidado la cita de Ana, es posible advertir un elemento inquietante. Luego de nombrar algunas opciones alternativas de evaluación de los libros, concluye diciendo: "pero generalmente se usa evaluación", en alusión implícita a la prueba escrita, de la que ya había hablado. Con esta frase, tan representativa de una concepción como lapidaria en un contexto de innovación, la docente da cuenta de una concepción de evaluación en la que la prueba escrita es el único instrumento válido, mientras que las otras posibilidades mencionadas serían meras actividades que no pueden ni deben ser consideradas como verdaderas evaluaciones.

Para profundizar un poco más en el enfoque evaluativo de cada entrevistada, se preguntó acerca de los criterios que orientan la confección de los instrumentos de evaluación del PLC que, como ya hemos visto, corresponden mayoritariamente a la prueba. Debemos consignar que no fue una pregunta asimilada fácilmente, ya que todas las profesoras se manifestaron dubitativas e incluso desconcertadas ante su formulación. Se trata de la misma reacción constatada ante la pregunta inicial sobre la definición de la lectura literaria. De esta manera, es posible inferir que las docentes tampoco cuentan con un discurso elaborado sobre el rol de la evaluación de la lectura literaria y que tampoco hay criterios explícitos y establecidos que orienten su práctica evaluativa en el marco del PLC.

Optar por la prueba escrita, prácticamente como único instrumento de evaluación de la lectura literaria, implica una serie de consideraciones por defecto (por decirlo de alguna manera), que dan cuenta de los criterios evaluativos de las docentes, aunque estas no lo hayan hecho explicito en sus respuestas. Así, siguiendo los criterios establecidos por Colomer (2005, a partir de Cairney, 1992) para el trabajo con los libros de literatura, al inclinarse por la prueba significa, al menos: (1) se privilegia el trabajo individual de los estudiantes por sobre el trabajo cooperativo de interpretación del texto; y (2) se trabajar en base a un formato único, impidiéndole a los alumnos seleccionar la modalidad que les sea más cómoda para establecer un diálogo con el libro. 
Los otros criterios rescatados en el marco de referencia, y que no hemos mencionado, dicen relación con el desarrollo de análisis globales de los textos y con tareas de interpretación abierta que valoren las distintas reflexiones suscitadas (no de respuesta única). Dentro de las posibilidades de la prueba como instrumento de evaluación, la adopción de estos dos últimos criterios es factible; sin embargo, su consideración va a depender en último término tanto de las creencias y representaciones sobre la lectura literaria del docente como del concepto de evaluación que maneje. Al revisar la información recopilada respecto a los criterios que guían la construcción de la prueba, surgieron ideas como las siguientes:

Medir si realmente comprendieron lo leído, no solamente a nivel literal, explícito, sino que, más que nada, uno apunta a la inferencia (Ana).

Pruebas objetivas con preguntas abiertas y de selección múltiple, donde incluye términos pareados, completación y todo eso (Teresa).

De acuerdo al libro leído, de acuerdo al grupo... si es un grupo más grande [se refiere a la edad], a lo mejor va a tener un nivel más de comprensión más amplio (...) En realidad cuando yo escojo los libros, me preocupo bastante de la parte valórica, entonces... cosa que en algún minuto ellos se pongan en el lugar del personaje, cuál les gustaría ser, el por qué, qué es lo bueno y lo malo que hizo tal o cuál personaje. Entonces, más que nada en base a eso me preparo para hacer las preguntas. Eso es a lo que le doy mayor énfasis, pero trato... nunca hago pruebas terriblemente largas, porque el libro igual sigue en clases, le seguimos dando vueltas... (Teresa).

...yo voy viendo distintos tipos de habilidades dentro de la prueba. Por ejemplo, la primera parte es de "completo", donde el niño... la información necesaria, después "respondo" que es como desarrollo, después una parte de opinión donde el niño manifiesta su opinión, otra de deducción o conclusión o inferencia a veces, depende, y otra de relato donde tiene que contar. Entonces ahí uno ve como está el niño en descripción, en opinión, en... es toda una gama, hay algunas en que agrego orden cronológico. Por lo general esos son los ítems que tienen mis pruebas (Carmen).

Netamente, los criterios que yo uso son respecto a las habilidades lectoras y también con respecto a las habilidades de producción de texto. Yo me fijo harto en los mapas de progreso, tomo eso y con eso elaboro yo mis instrumentos. Fielmente yo pesco el mapa de progreso y esos son mis criterios, los que orientan... y a eso yo le sumo por ejemplo (...) pero el criterio base, básico es el mapa de progreso (Carmen).

Podríamos decir que, en su conjunto, las docentes relacionan la creación de sus instrumentos evaluativos con cuatro ámbitos. El más atendido y compartido por las entrevistadas es la diversificación tanto de los niveles de comprensión lectora a los cuales atienden las preguntas como de las habilidades que buscan promover con la evaluación. Señalan, por tanto, que dentro de las pruebas consideran el desarrollo de baterías de preguntas que apuntan a los tres niveles de comprensión: literal, inferencial y crítico/ valorativo, remarcando, en el caso de Ana, que para observar si los estudiantes comprendieron lo leído, hace falta atender no solamente al nivel literal.

Hacer pruebas que enfaticen en "la parte valórica" de los textos, en el caso de Teresa, atender a las características del grupo de estudiantes para el cual se realiza la evaluación, y que los instrumentos creados respondan a los Mapas de Progreso ${ }^{9}$ del Ministerio de

8 Los Mapas de Progreso son descripciones de la progresión de los aprendizajes en los diferentes niveles educativos y áreas curriculares, realizados por la Unidad de Currículum y Evaluación del Ministerio de 
Educación, son otros de los criterios surgidos en las entrevistadas como orientadores para la confección de las pruebas del PLC, aun cuando, como decíamos, la primera reacción de las docentes frente a esta pregunta haya sido de indefinición y de duda.

\subsection{LA INFORMACIÓN PROVENIENTE DE LOS INSTRUMENTOS DE EVALUACIÓN UTILIZADOS}

Con el fin de contrastar empíricamente la información recogida en las entrevistas, recurrimos también a una muestra de instrumentos de evaluación proporcionados por las docentes que, como se ha dicho inicialmente, correspondió a 3 pruebas por cada una de ellas, sumando un total de 9 instrumentos para el análisis. Las pruebas corresponden a los siguientes libros: "Historia de una gaviota y del gato que le enseñó a volar", "Aventuras de un niño de la calle", "La abuela", "El misterio de los piñones", "Mundo flotante", "Pregúntale a Alicia", "El Principito", "La nariz de Moritz" y "La historia de Manu".

El procedimiento de análisis, en este caso, estuvo basado en la búsqueda de frecuencias, a partir de la consideración de los ítems y preguntas incluidos en las pruebas en cuanto al nivel de comprensión lectora (literal, inferencial o crítico) al que apuntaban. En las siguientes tablas se presentan los resultados sistematizados:

\begin{tabular}{|c|c|c|}
\hline Niveles & Frecuencia & \% \\
\hline Literal & 123 & 72,8 \\
\hline Inferencial & 26 & 15,4 \\
\hline Crítico & 20 & 11,8 \\
\hline Total & 169 & 100 \\
\hline
\end{tabular}

Tabla 1. Clasificación preguntas según niveles de comprensión

$\mathrm{Al}$ analizar los instrumentos de manera conjunta, es posible observar que el tipo de pregunta predominante apunta a un nivel de comprensión literal de los textos: prácticamente 3 de cada 4 de los 169 ítems observados corresponde a preguntas de reconocimiento literal, es decir, de recuerdo de situaciones, personajes, trama, etc. Mientras, un reducido porcentaje (15\% del total) apela a hacer inferencias sobre lo leído, y un número aún más reducido se dirige a la apreciación crítica y valorativa de los estudiantes sobre lo leído, cifra que supera escasamente el $10 \%$ del total de preguntas.

Asimismo, resulta interesante observar estos datos agrupándolos de acuerdo al nivel educativo al cual está dirigida cada una de las pruebas, operación tras la cual se obtiene la siguiente distribución en términos de frecuencias y porcentajes:

\begin{tabular}{|c|c|c|c|c|}
\hline \multirow{2}{*}{ Niveles } & \multicolumn{2}{|c|}{ Instrumentos $5^{\circ}$ y $6^{\circ}$ básico } & \multicolumn{2}{c|}{ Instrumentos $7^{\circ}$ y $8^{\circ}$ básico } \\
\cline { 2 - 5 } & Frecuencia & $\%$ & Frecuencia & $\%$ \\
\hline Literal & 96 & 81,4 & 27 & 52,9 \\
\hline Inferencial & 11 & 9,3 & 15 & 29,4 \\
\hline
\end{tabular}

Educación de Chile. En el caso de Lenguaje y Comunicación, los hay en Lectura y Producción de Textos. 


\begin{tabular}{|c|c|c|c|c|}
\hline Crítico & 11 & 9,3 & 9 & 17,6 \\
\hline Total & 118 & 100 & 51 & 100 \\
\hline
\end{tabular}

Tabla 2. Clasificación preguntas según niveles de comprensión, diferenciado por curso

Tal como se puede apreciar, la agrupación de los instrumentos por nivel educativo muestra diferencias significativas entre unos cursos y otros. El porcentaje de interrogantes que apuntan al nivel literal de los textos es visiblemente superior en el caso de los instrumentos destinados a evaluar lecturas en $5^{\circ}$ y $6^{\circ}$ básico, mientras que las preguntas de tipo inferencial y crítico aumentan significativamente su presencia en los instrumentos dirigidos a $7^{\circ}$ y $8^{\circ}$ básico. En otras palabras: por una pregunta inferencial que se haga en $5^{\circ}$ ó $6^{\circ}$ básico, se hacen 3 en $7^{\circ}$ u $8^{\circ}$; asimismo, entre uno y otro nivel se doblan las preguntas de sentido crítico y de valoración sobre el texto. Lo anterior se condice con la extendida -y errada- idea de que los niveles de lectura corresponden a una secuencia progresiva y escalonada de adquisición de los mismos, siendo que en verdad son diferentes puertas de entrada a los textos, que implican estrategias igualmente diferentes, pero complementarias, de acceso a la lectura.

Finalmente, es interesante observar los tipos de preguntas que realiza cada docente participante en el estudio, toda vez que en las entrevistas hemos intentado acceder a las concepciones que cada una tiene sobre el hecho literario y sobre la evaluación del PLC, para ver posibles relaciones entre esas concepciones y los instrumentos evaluativos que utiliza. Cabe recalcar que aunque las muestras no están homologadas, los datos obtenidos nos permiten hacernos una idea al respecto, tal como se observa en la siguiente tabla:

\begin{tabular}{|c|c|c|c|c|c|c|}
\hline \multirow{2}{*}{ Niveles } & \multicolumn{2}{|c|}{ Ana } & \multicolumn{2}{c|}{ Teresa } & \multicolumn{2}{c|}{ Carmen } \\
\cline { 2 - 7 } & Frecuencia & $\%$ & Frecuencia & $\%$ & Frecuencia & $\%$ \\
\hline Literal & 44 & 75,9 & 34 & 60,7 & 45 & 81,8 \\
\hline Inferencial & 9 & 15,5 & 14 & 25 & 3 & 5,5 \\
\hline Crítico & 5 & 8,6 & 8 & 14,3 & 7 & 12,7 \\
\hline Total & 58 & 100 & 56 & 100 & 55 & 100 \\
\hline
\end{tabular}

Tabla 3. Clasificación preguntas según niveles de comprensión, diferenciado por docente

De acuerdo a los resultados obtenidos, si bien no se producen diferencias abismantes entre una y otra docente, la proporción en la que se presentan las preguntas revela matices interesantes. De todas, quien muestra un mayor porcentaje de interrogantes de tipo apreciativas o críticas es Teresa, con un porcentaje cercano al $15 \%$ del total; le sigue Carmen con un 12,7\%; y luego Ana con $8.6 \%$. Si nos detenemos en las preguntas de tipo inferencial, nuevamente es Teresa quien lidera el grupo, con un 25\%; luego Ana con un $15 \%$; y Carmen con un $5.5 \%$.

$\mathrm{Si}$ volvemos a las citas expuestas anteriormente, respecto a los criterios que orientan la creación de los instrumentos, podemos observar algunos pasajes que refuerzan los resultados obtenidos. Por ejemplo, en el caso de Teresa se menciona explícitamente la preocupación por introducir preguntas de tipo valorativo que, aun cuando estén muy 
influenciadas por un eje de valoración principalmente moral, tienen el propósito de que los estudiantes sean capaces de desarrollar argumentos y opiniones frente al texto leído:

En realidad cuando yo escojo los libros, me preocupo bastante de la parte valórica, entonces... cosa que en algún minuto ellos se pongan en el lugar del personaje, cuál les gustaría ser, el por qué, qué es lo bueno y lo malo que hizo tal o cuál personaje. Entonces, más que nada en base a eso me preparo para hacer las preguntas.

Estas definiciones de Teresa sobre la valoración personal de las lecturas se complementan con el mayor porcentaje de preguntas de tipo inferencial observado en este estudio: un $25 \%$ de sus ítems, lo que, en conjunto, hace que su "dependencia" del nivel textual sea bastante menor que el promedio: un 60\%, mientras las otras profesoras superan las barreras del $75 \%$ y $80 \%$. En el caso de Carmen, también es posible vislumbrar en sus respuestas una alusión a incluir preguntas que apuntan a recoger la opinión de los estudiantes sobre lo leído:

...yo voy viendo distintos tipos de habilidades dentro de la prueba. Por ejemplo, la primera parte es de "completo", donde el niño... la información necesaria, después "respondo" que es como desarrollo, después una parte de opinión donde el niño manifiesta su opinión, otra de deducción o conclusión o inferencia a veces, depende, y otra de relato donde tiene que contar.

Ana, por su parte, no menciona el desarrollo de valoraciones críticas como parte de sus criterios orientadores, lo que se condice con tener el porcentaje más bajo de ítems asignados para este nivel de lectura (8\%), pero sí lo hace refiriéndose a las inferencias que busca promover: "más que nada, uno apunta a la inferencia". En ese sentido, llama la atención el bajo porcentaje de preguntas inferenciales que presentan sus instrumentos: solo un $15 \%$ de los ítems, muy poco si atendemos al carácter central que le otorga en su discurso.

En definitiva, si bien se observan ciertos matices entre uno y otro caso, el contexto general de las tres entrevistadas es el de un discurso basado, como ya adelantáramos, en la diversificación de los tipos de preguntas realizadas sobre las lecturas. En este marco, causa sorpresa el poco espacio que las docentes otorgan a los niveles inferencial y valorativo, a favor de una presencia más constante y definitiva de las preguntas de orden literal que, en su conjunto, suman más del $72 \%$ de los ítems que debieron responder los estudiantes luego del proceso de lectura de las obras literarias en cuestión.

Una línea explicativa para lo anterior sería que esos discursos, más que asentarse verdaderamente en sus creencias sobre la evaluación de la comprensión en la lectura literaria, estarían relacionados con los discursos oficiales que circulan en la cultura escolar acerca de la importancia de trabajar todos los niveles de comprensión de un texto. Seguir esta línea ayudaría a explicar la ausencia de equilibrio entre los diversos ítems, toda vez que los discursos oficiales no afectan tan profundamente las prácticas didácticas del profesorado como sí lo hacen sus concepciones y creencias individuales.

\section{CONCLUSIONES}

Finalizada esta pequeña investigación, hemos constatado lo provechoso que ha sido adentrarse en un terreno que, como señalábamos al inicio, ha sido y es foco de dificultades en el contexto de la educación literaria de los niños y, particularmente, de adolescentes y 
jóvenes. En el intento por sistematizar los hallazgos y revelaciones surgidos del proceso, destacaremos como puntos relevantes los siguientes:

1. Respondiendo a nuestra pregunta de investigación, señalaremos que los resultados confirman la existencia de un hilo que relaciona las prácticas didácticas del profesorado con sus sistemas de creencias, representaciones y saberes sobre el objeto de enseñanza, tal como lo viene demostrando la investigación sobre el pensamiento del profesor. Con respecto al tipo de relación establecida, en nuestro caso hemos constatado que concepciones más o menos empobrecidas conceptual y teóricamente sobre lo que significa leer literatura y sobre los aprendizajes que están en juego en este tipo de lectura, devienen en prácticas evaluativas "opacas" en el contexto del Plan de Lectura Complementaria, que dan muy poco de sí en el contexto de la educación literaria de las actuales generaciones. Una concepción basada fundamentalmente en el tópico genérico de la importancia de generar hábitos lectores, que no profundiza en la especificidad de los textos literarios ni en la importancia de adquirir y hacer progresar una competencia de lectura igualmente específica para estos, tiene directa relación con dos decisiones didácticas que suele tomar el profesorado en el contexto del PLC. La primera es decidir (en el entendido de que no está obligado a ello) que la mejor manera de dar cuenta de un proceso de lectura literaria es una prueba escrita, individual y estándar para todos. La segunda, que en esa prueba escrita debe primar la capacidad del estudiante para recordar la historia, mucho más que la construcción (idealmente colectiva, como lo sería en una verdadera "comunidad de lectores") de significados sobre lo leído. Una posible línea explicativa para lo anterior estaría en la extensa "tradición" que exhibe esta práctica evaluativa en el contexto educacional chileno: probablemente las docentes vivieron esa prueba escrita en su propia etapa escolar, no la cuestionaron en sus procesos de formación inicial, y la han seguido viviendo en sus diversos entornos de trabajo. Creemos, no obstante, que la explicación se completa con la indeterminación sobre el lugar que ocupa la literatura en la clase de Lenguaje y Comunicación, pues la pervivencia de prácticas como estas nos llevan a pensar en la ausencia de una reflexión seria sobre cómo progresa el aprendizaje lector y, particularmente, la competencia de lectura literaria de los estudiantes.

2. La concepción de la evaluación continúa muy apegada a su función de certificación de la realización de una actividad determinada (en nuestro caso, de lectura de un libro en particular), pues dados los datos aquí recogidos no podríamos hablar de un proceso de aprendizaje de ciertas estrategias de entrada a los textos que favorezcan la progresiva adquisición de la competencia literaria. Así, la visión tradicional de la prueba como "comprobación" de que efectivamente se ha leído (como si el aprendizaje lector estuviese en la ejecución misma de la acción) sigue siendo la perspectiva predominante a la hora de pensar las prácticas evaluativas relacionadas con la lectura de literatura. Lejos todavía de la concepción actual de evaluación formativa, que implica la integración de las instancias evaluativas en el transcurso de los procesos de aprendizaje y la consideración de una función reguladora que, en la perspectiva de Allal, define todos los procesos evaluativos (y en definitiva, todos los procesos de aprendizaje), la evaluación en el marco de los Planes de Lectura Complementaria 
cumple una función que la aleja de su papel orientador del aprendizaje, pasando a ser el objetivo en sí misma que toda la investigación educativa actual ha denunciado.

3. Tal como ya ha señalado la investigación en nuestro campo, que habla de un promedio de una pregunta de comprensión lectora inferencial por cada cinco literales, creemos que los resultados aquí expuestos adscriben a una problemática cada vez más evidente en la didáctica de lengua y literatura: el tipo de lector que serán nuestros niños y jóvenes depende, en buena medida, del lector que construimos en nuestras prácticas didácticas. Así, alumnos con muy pocas experiencias de lectura inferencial presentarán importantes problemas a la hora de responder pruebas estandarizadas que focalizan en este tipo de lectura (como les sucede a los escolares chilenos). Del mismo modo, a estudiantes cuya experiencia con la literatura se reduce, para muchos de ellos, a leer mensualmente un libro y contestar preguntas sobre lo que a juicio del profesor debiera recordar de esa historia, es difícil pedirles (menos exigirles, como hacen las sociedades actuales) hábitos permanentes de lectura e incluso que sean capaces de realizar una lectura fruitiva de la literatura que llega a sus manos, como también se les suele exigir bajo las banderas del "goce" de la lectura.

4. Aunque no formaba parte de la formulación inicial del proyecto, la presente investigación ha confirmado nuestras sospechas sobre el carácter autónomo y escasamente integrado del PLC en el área de Lenguaje y Comunicación. La ausencia de objetivos y de criterios que orienten la construcción de un verdadero itinerario de lecturas literarias complementarias a las del devenir cotidiano del aula (reafirmada por el desconcierto de las docentes frente a una pregunta que indague en estos puntos), hace que el plan lector sea percibido -incluso por el profesorado- como una instancia aislada y, sobre todo, sin un sentido más allá del tener que cumplir con las "exigencias" del sistema, como lo llamó una de las entrevistadas, exigencia que avanza por dos cauces: tanto en el deber colocar una calificación por esta actividad ("evaluarla", en su sentido más restringido) como en el deber de prescribir cierto número de obras que sean obligatorias para todos. Si esta es la percepción de las docentes sobre el PLC, a nadie extrañará que la visión de los estudiantes relacione estas lecturas con un "leer porque hay que leer", sin ninguna motivación o desafío que no sea el contestar una prueba basada mayoritariamente en el recuerdo de la historia.

Creemos, en definitiva, en la importancia de hacer investigaciones que hagan avanzar los conocimientos existentes en el campo de la educación literaria sobre los planes de lectura complementaria que, quizás bajo otros nombres o formulaciones, suelen existir en las escuelas de muchos países y contextos educativos. ¿Qué sabemos sobre este "formato" de lectura, que tanto parece haber afectado la motivación del alumnado hacia la literatura? ¿Cómo se integra a los procesos de aprendizaje literario de nuestros estudiantes? ¿Qué opinión les merece a los alumnos?,+ ¿Qué obras se incluyen y bajo qué criterios? ¿Cómo se evalúa y qué motivos nos llevan a evaluarlo de esa manera? Son algunas de las preguntas que creemos relevantes en este ámbito y con las cuales el presente documento pretende dialogar. 


\section{REFERENCIAS BIBLIOGRÁFICAS}

Allal, L. (2006). Une conceptualisation de l'évaluation dans la recherche francophone en Europe. En G. Figari \& L. Mottier, Recherche sur l'évaluation en éducation. París: L’Harmattan.

Bordons, G. \& Díaz Plaja, A. (2004). Introducció. En G. Bordons \& A. Díaz Plaja (Coords.), Ensenyar literatura a secundària (pp. 7-16). Barcelona: Graó.

Briz Villanueva, E. (2003). La evaluación de las habilidades lingüísticas. En A. Mendoza (Coord.), Didáctica de la Lengua y la Literatura (pp. 425-465). Madrid: Pearson.

Briz Villanueva, E. (1998). La evaluación en el área de lengua y literatura. En A. Mendoza (Coord.), Conceptos clave en Didáctica de lengua y la literatura (pp. 115-140). Barcelona: SEDLL/ ICE-UB/Horsori.

Cambra, M. (2000). Introducció. El pensament del professor: formació per a la pràctica reflexiva. En A. Camps, I. Ríos \& M. Cambra (Coord.), Recerca i formació en didàctica de la llengua (pp. 161-172). Barcelona: Graó.

Català, G. et al. (2001). Evaluación de la comprensión lectora. Barcelona: Graó.

Colomer, T. (2009). L'escola fa o des fa lectors. Faristol, n. 63, 8-10.

Colomer, T. (2005). Andar entre libros: la lectura literaria en la escuela. México DF: Fondo de Cultura Económica.

Colomer, T. (1998). L'ensenyament de la lectura. En A. Camps \& T. Colomer (Coords.), L'ensenyament $i$ l'aprenentatge de la llengua i la literatura en l'educació secundaria (pp. 49-68). Barcelona: ICE/UB- Horsori.

Colomer, T. (1996). La didáctica de la literatura: temas y líneas de investigación e innovación. En C. LOMAS (Coord.), La educación lingüística y literaria en la educación secundaria (123142). Barcelona: ICE/UB- Horsori.

Colomer, T. \& Margallo, A.M. (s/f). L'aprenentatge de la comprensió. Documento de trabajo, sin publicar.

Marcelo García, C. (1987). El pensamiento del profesor. Barcelona: CEAC.

Mendoza, A. (2000). L'elaboració d'un instrument per a la regulació formativa del aprenentatges. Descripció d'un projecte d'avaluació per a l'àrea de llengua i literatura. En A. Camps, I. Ríos \& M. Cambra (Coords.), Recerca i formació en didàctica de la llengua (pp. 117-134). Barcelona: Graó.

Palou, J. et al. (2000). Els professors de llengua: entre el desig i la realitat. En A. Camps, I. Ríos \& M. Cambra (Coord.), Recerca i formació en didàctica de la llengua (pp. 173-182). Barcelona: Graó.

Ribas, T. (2010). La evaluación en el área lingüística. Textos de Didáctica de la Lengua y de la Literatura, n. 53, 10-21.

Ribas, T. (1997) Introducció. En T. Ribas (coord.), L'avaluació formativa en l'àrea de llengua (pp. 5-12). Barcelona: Graó.

Sánchez Corral, L. (2003). Didáctica de la literatura: relaciones entre el discurso y el sujeto. En A. Mendoza (Coord.), Didáctica de la Lengua y la Literatura (pp. 291-317). Madrid: Pearson.

Sánchez Enciso, J. \& Rincón, F. (1987). Enseñar literatura. Barcelona: Laia.

SÁNCHEZ MIGUEL, E. (1997). L'avaluació formativa de la comprensió lectora. En T. Ribas (Coord.), L'avaluació formativa en l'àrea de llengua (pp. 83-107). Barcelona: Graó.

Tauveron, C. (2002). Lire la littérature à l'école. Paris : Hatier. 